\title{
Spatio-Spectral Measurement of a Surface Plasmon Polariton in a Gold Nano-Slit Array
}

\author{
Soobong ChOI, DooJae PARK and D. S. Kim* \\ Department of Physics and Astronomy, Seoul National University, Seoul 151-747 \\ M. S. JeOng and Clare C. ByeOn \\ Advanced Photonics Research Institute, GIST, Gwangju 500-712
}

(Received 13 May 2008)

\begin{abstract}
A spatio-spectral measurement of transmitted near field distribution from a nano optic structure is demonstrated by near field scanning optical miscroscopy (NSOM) with the point spectroscopic approach. This method provides spectral information of nano-optic system with sub-diffraction limit spatial resolution. The gold nano slit array is a good model system where analytical calculation is possible by the modal expansion method for all diffraction orders with surface impedance boundary conditions. The point spectroscopic analysis of near field distribution shows a good agreement with theoretical model calculation.
\end{abstract}

PACS numbers: 73.20.M, 07.79.Fc, 42.62.Fi

Keywords: Surface plasmon, Near-field scanning optical microscopy, Laser spectroscopy

\section{INTRODUCTION}

Since the observation of extraordinary optical transmission through subwavelength hole arrays [1], considerable theoretical and experimental efforts have been devoted to interpreting the essential physics of the process in metal nano apertures $[2-5,17]$. Especially, many intriguing properties of metallic diffraction gratings involve the excitation of surface plasmon polariton waves. Surface plasmon polaritons (SPP) are surface-bound waves at the metal-dielectric interface, owing their existence to surface charges. It has been theoretically shown that the spatial position of the maximum field enhancement in inhomogeneous nano-systems can be controlled using the broadband femtosecond pulses [6-10]. Femtosecond evolution of localized hot spots and resonant surface plasmons in mesa-structured Ag gratings [8] and on circular disk arrays [9] has been coherently controlled by using ultrafast excitation pulses. Though these results show some dramatic change in the near-field distribution, they give information only about the temporal evolution of the system. Experimentally, direct measurement of transients at a hot spot is not an easy task because the hot spot size is far below the diffraction limit of the probing wavelength. On the other hand, temporal information on SPPs can be understood more easily via a spectral analysis of the near-field distribution and its Fourier analysis into the time domain.

*E-mail: dsk@phya.snu.ac.kr; Fax: +82-2-884-3002
In this paper, we have studied a nano-optical model system for plasmonic femtosecond excitation by using point spectroscopy with a near-field scanning optical microscope (NSOM), in which the experimental results are essentially understandable in an analytical way. In onedimensional periodic nanoslit arrays, an accurate modeling of near-field patterns, including their spatio-spectral dependencies, is possible when using a diffraction order expansion method together with the surface impedance boundary matching condition (SIBC) $[1,11,12]$. In this model, the results can provide full spatio-spectral information on the nano-optic system despite the diffraction limit of the excitation wavelength. We successfully demonstrate the point spectroscopic measurement of the near-field distribution, reflecting the wavelengthdependant localization of SPP fields, by using femtosecond pulses with the measurements being in close agreement with the predictions from model calculations.

\section{SURFACE PLASMON POLARITON IN GOLD NANO SLIT ARRAY}

To measure the spatio-spectral near-field distribution, we built a NSOM with a spectrometer as shown in Figure 1. The near-field distributions were collected by using an apertured NSOM tip and were analyzed by using a spectrometer with a CCD detector. To make point spectroscopic measurements, we synchronized the movement of NSOM tip position with the CCD acquisition timing. 


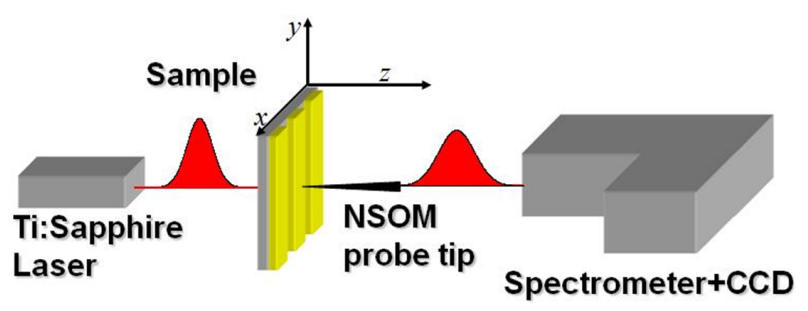

Fig. 1. Schematic diagram of point spectroscopy with a near-field scanning optical microscope. An aperture-type NSOM tip collects the near-field distribution and sends the optical signal to the spectrometer for analyzing. (a)

(b)

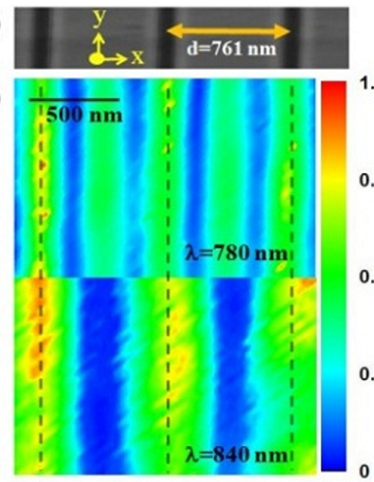

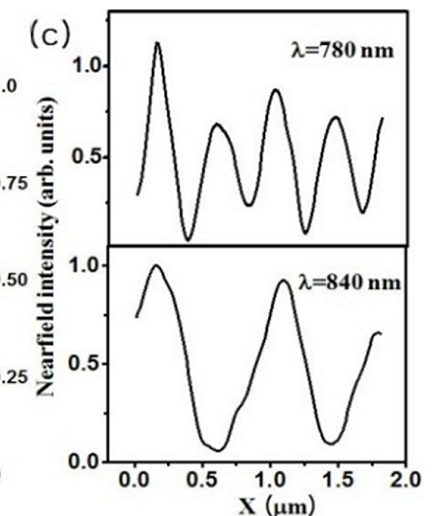

Fig. 2. (a) Scanning electron microscope image of the nanoslit array. (b) Near-field transmission images of the nanoslit array excited by monochromatic light with a wavelength of $780 \mathrm{~nm}$ (top) and $840 \mathrm{~nm}$ (bottom). The slit positions are depicted as black dashed lines in (b). (c) Cross sections of (b) showing the double-periodic and the singleperiodic patterns within one lattice constant for excitation wavelengths of $780 \mathrm{~nm}$ (top) and $840 \mathrm{~nm}$ (bottom) respectively.

Figure 2(a) shows the studied metallic nanoslit array which has a period $d=761 \mathrm{~nm}$ and a slit width of 100 $\mathrm{nm}$. This nanoslit array was fabricated by using electron beam lithography, followed by ion beam milling, on an 80 -nm-thick gold film evaporated onto a $350-\mu$ m-thick sapphire substrate. In this structure, SPP modes can be resonantly excited by TM polarized light through grating coupling at either the air-metal or the sapphire-metal interfaces. The resonances are characterized by the grating momentum $n \mathrm{G}(n= \pm 1, \pm 2, \pm 3, \ldots)$ with $\mathrm{G}=2 \pi / \mathrm{d}$, which is transferred to the incident light. The experiments were performed with laser pulses centered around the lowest order $\mathrm{AM}[ \pm 1]$ resonance at the air-metal interface at $\lambda_{S P}=d \sqrt{\varepsilon_{m} /\left(\varepsilon_{m}+1\right)} \approx 780 \mathrm{~nm}$ [11-13]. Here, $\varepsilon_{m}$ is the real part of the dielectric constant of gold. For a narrow slit, the coupling efficiencies are the same for all diffraction orders, which is termed as the equipartition of the diffraction order $[11,18]$. However, at the SPP resonance, this equipartition of the diffraction orders forces suppression of all other modes, including the $0^{\text {th }}$ order, except for the +/-1 SPP modes. In a simplified sense, (a)

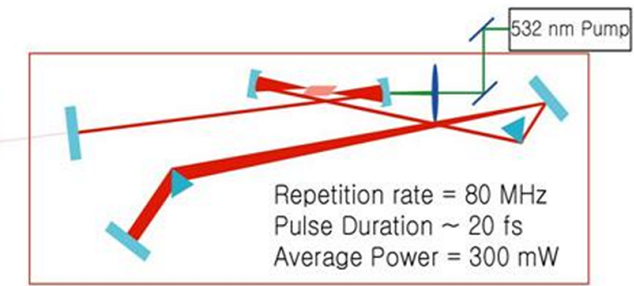

(b)

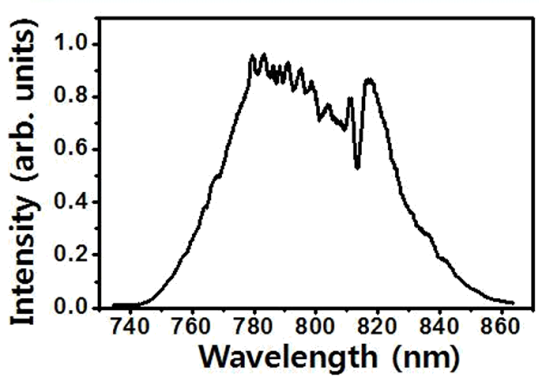

Fig. 3. (a) Schematic of the Ti:Sappire oscillator used in our experiment. The oscillator produces a pulse train at 80 $\mathrm{MHz}$ repetition rate and the time duration of each pulse is about $20 \mathrm{fs}$. The average power is $300 \mathrm{~mW}$. (b) The spectrum of femtosecond laser pulses in the wavelength range from 750 $\mathrm{nm}$ to $850 \mathrm{~nm}$ for our experiment.

we may consider only the $0^{t h}$ and the $1^{\text {st }}\left(T_{0}\right.$ and $T_{1}$, respectively) diffraction orders at the expense of all higher diffraction orders, which are much more weakly excited.

We can describe the time-integrated near-field intensity as a function of position:

$$
\begin{gathered}
I(x) \cong \int_{-\infty}^{\infty}\left|\exp (-i \omega t)\left(T_{0}+T_{1} e^{i k_{l} x}+T_{1} e^{-i k_{l} x}\right)\right|^{2} d t \\
\cong\left\{\begin{array}{lc}
2\left|T_{1}\right|^{2}\left(1+\cos 2 k_{1} x\right) ; & T_{0} \ll T_{1} \\
\left|T_{0}\right|^{2}+2\left(T_{0} T_{1}^{*}+T_{0}^{*} T_{1}\right) \cos k_{1} x ; & T_{0} \gg T_{1}
\end{array}\right.
\end{gathered}
$$

The sign of $k_{1}$ represents the direction of SPP wave propagation on the sample surface. In the on-resonant SPP coupling case, $T_{1}$ is dominated by equipatition of the diffraction order theorem, leading to $\cos 2 k_{1} x$ as in Eq. (1). The period of this standing wave pattern is only half the original slit period, an unambiguous signature of SPP excitation. On the contrary, in the offresonance $\mathrm{SPP}$ coupling case, $T_{0}$ is dominant, leading to $\cos k_{1} x$ as in Eq. (1). Figure 2(b) shows the near-field intensities, $I(x, y)$, when monochromatic waves of $780 \mathrm{~nm}$ (SPP on-resonance, top) and $840 \mathrm{~nm}$ (SPP off-resonance, bottom) are incident on the nanoslit array. The slit position is depicted as a grey dashed line for each case. In Figure $2(\mathrm{c})$, the standing wave pattern $\left(\cos 2 k_{1} x\right.$ in (1)), whose period is half the slit period is observed at the SPP resonance (top), while the bottom shows single-periodic illumination ( $\cos k_{1} x$ in Eq. (1)) with respect to the slits off-resonance.

In the spectral domain, the interference between these two contributions gives rise to characteristic asymmetry, "Fano-like" line shapes in the transmission [14]. These results suggest that the near-field distributions can be 

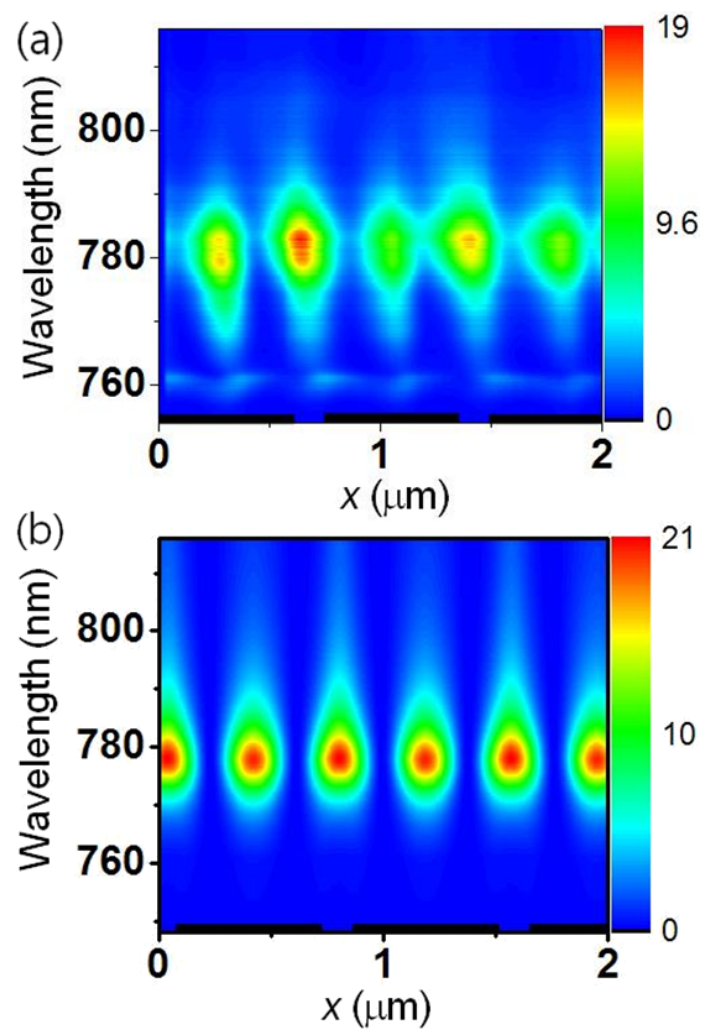

Fig. 4. Spatio-spectral near-field image of a nano-slit array. (a) Experimental data from point spectroscopic NSOM. (b) Theoretical calculation using the modal expansion method with SIBC. The sample structures are depicted as black solid lines in (a) and (b).

varied by using the wavelength of the excitation light. Thus, in order to know the complete spectral response of each position on the nano slit arrays, one must investigate all wavelength cases. In our experimental condition, the nano-slit array was designed to have the resonant SPP at the $780 \mathrm{~nm}$, as mentioned above. For this reason, broadband femtosecond laser pulses which covered the wavelength range from $750 \mathrm{~nm}$ to $850 \mathrm{~nm}$ were used. Figure 3 shows the schematics of the femtosecond laser system. The pulses were externally compensated by a pair of negative-group-velocity compensation mirrors and a pulse duration of about 20 fs at the sample position was achieved. The sample was illuminated from the substrate side with TM polarized pulses (E-fields perpendicular to the slit direction).

For pulsed excitation, the Fourier amplitude, $t_{n}(\omega)$, for the $\mathrm{n}^{t h}$-order diffraction order is weighted by $S(\omega)$, which is the spectral composition of the excitation pulses. Thus, the near-field distribution as a function of position is described as follows:

$$
\begin{array}{r}
I(x)=\mid \sum_{n=0}^{\infty} \int_{0}^{\infty}\left\{t_{n}(\omega) \exp \left(i k_{n} x\right)\right. \\
\left.+t_{n}(\omega) \exp \left(-i k_{n} x\right)\right\}\left.S(\omega) d \omega\right|^{2}
\end{array}
$$

To compare the simulations, which have been performed for all vector components of the electromagnetic field, to experiment, one needs additional information about the polarization-sensitive collection properties of the fiber probe aperture. A circularly-apertured fiber tip has been reported to collect the in-plane electric field component $E_{x}$ perpendicular to the slit axis and magnetic field $H_{y}$ along the slit axis with approximately the same efficiency $[13,15,16]$. We therefore assumed an equal coupling ratio for $E_{x}$ and $H_{y}$ such that the collected intensity was proportional to $\left|E_{x}\right|^{2}+\left|H_{y}\right|^{2}$.

Figure 4(a) shows the spatio-spectral near-field distribution in the nanoslit array and (b) shows the theoretical simulation using the modal expansion method with SIBC. The experimental result shows a good agreement with the theoretical result. At the resonant SPP generation wavelength $(\lambda=780 \mathrm{~nm})$, the measured near-field distribution shows a clear standing wave pattern where period is half the original slit period, as explained in Figure 2. The femtosecond excitation of SPP fields is clearly established and in the region between the slits, the SPP field is localized to about $200 \mathrm{~nm}$, which is, indeed, far below the diffraction limit $(\sim 400 \mathrm{~nm})$ of the excitation pulses.

\section{CONCLUSION}

In conclusion, we have demonstrated the experimental measurement of the wavelength dependant spatiospectral near-field distribution of a nano-slit array with sub-diffraction limit resolution by using a point spectroscopic NSOM with broadband femtosecond laser excitation which could not be measured by using a classical far-field measurement due to the evanescent nature of the SPP field. The measured near-field distribution in metal nano-slit array shows resonant behavior at $\lambda_{s p}(=780 \mathrm{~nm}$ in our experiment), which is determined by the lowestorder $\mathrm{AM}[ \pm 1]$ resonance at the air-metal interface. The measurement also shows a clear near field confinement at the region between the slit's position $(\sim 200 \mathrm{~nm})$ at the resonant SPP excitation wavelength. We believe that our results will pave the way for the future development of nano optical applications through spatio-spectral characterizations by using a NSOM with broadband femtosecond pulse excitation.

\section{ACKNOWLEDGMENTS}

The authors thank Korea Government (Ministry of Science and Technology, Ministry of Education \& Human Resources Development) for financial support through Korean Research Foundation (grant number C00012 and C00032), Korean Science and Engineering Foundation, the Seoul R\&BD program and the Nano R\&D program (Grant number 2007-02939). 


\section{REFERENCES}

[1] H. Lochbihler, Phys. Rev. B 50, 4795 (1994).

[2] T. W. Ebbesen, H. J. Lezec, H. F. Ghaemi, T. Thio and P. A. Wolff, Nature 391, 667 (1998).

[3] H. J. Lezec, A. Degiron, E. Devaux, R. A. Linke, L. Martin-Moreno, F. J. Garcia-Vidal and T. W. Ebbesen, Science 297, 820 (2002).

[4] P. G. Kik, S. A. Maier and H. A. Atwater, Phys. Rev. B 69, 045418 (2004).

[5] H. F. Schouten, N. Kuzmin, G. Dubois, T. D. Visser, G. Gbur, P. F. A. Alkemade, H. Blok, G. W.'t Hooft, D. Lenstra and E. R. Eliel, Phys. Rev. Lett. 94, 053901 (2005).

[6] M. I. Stockman, S. V. Faleev and D. J. Bergman, Phys. Rev. Lett. 88, 067402 (2002).

[7] M. I. Stockman, D. J. Bergman and T. Kobayashi, Phys. Rev. B. 69, 054202 (2004).

[8] A. Kubo, K. Onda, H. Petek, Z. Sun, Y. S. Jung and H. K. Kim, Nano Lett. 5, 1123 (2005).

[9] M. Aeschlimann, M. Bauer, D. Bayer, T. Brixner, F. J. G. D. Abajo, W. Pfeiffer, M. Rohmer, C. Spindler and F. Steeb, Nature 446, 301(2007).
[10] A. Kubo, N. Pontius and H. Petek, Nano Lett. 7, 470 (2007).

[11] K. G. Lee and Q. H. Park, Phys. Rev. Lett. 95, 103902 (2005).

[12] C. Ropers, D. J. Park, G. Stibenz, G. Steinmeyer, J. Kim, D. S. Kim and C. Lienau, Phys. Rev. Lett. 94, 113901 (2005).

[13] D. J. Park, S. B. Choi, K. J. Ahn, D. S. Kim, J. H. Kang, Q.-H. Park, M. S. Jeong and D.-K. Ko, Phys. Rev. B. 77, 115451 (2008).

[14] C. Genet, M. P. van Exter and J. P. Woerdman, Opt. Comm. 225, 331 (2003).

[15] C. Obermuller and K. Karrai, Appl. Phys. Lett. 67, 3408 (1995).

[16] K. G. Lee, H. W. Kihm, J. E. Kihm, W. J. Choi, H. Kim, C. Ropers, D. J. Park, Y. C. Yoon, S. B. Choi, D. H. Woo, J. Kim, B. Lee, Q. H. Park, C. Lienau and D. S. Kim, Nat. Photon. 1, 53 (2007).

[17] D. H. Hurley and R. Lewis, J. Korean Phys. Soc. 51, 368 (2007).

[18] Q.-H. Park, K. G. Lee and D. S. Kim, J. Korean Phys. Soc. 49, 2075 (2006). 